Editorial

\title{
Stevia rebaudiana bertoni as a natural sweetener
}

\section{Editorial}

It is widely acknowledged that increased consumption of sugar has resulted in several nutritional and medical problems including obesity. Historically, low caloric sweeteners have been investigated as a possible substitute for sugar; one important class of low caloric sugar substitutes is known as a high intensity sweetener which is at least 50-100times sweeter than sucrose. Nowadays, the most common high intensity sweeteners in the world market are made of synthetic compounds. A frequent metallic aftertaste of such synthetic sweeteners does not provide the realistic taste of sugar. In addition high concentrations of some types of synthetic sweeteners, such as saccharin, have been reported as being hazardous to health. Early studies on laboratory rats were found to relate specifically to the unique combination of high $\mathrm{pH}$, high calcium phosphate, and high protein levels in rat urine. Therefore, Saccharin induced may not be relevant to any risk to human health. ${ }^{1-3}$ On the other hand, reports published by The European Food Safety Authority ${ }^{4}$ indicate that low concentrations of low-calorie sweeteners are safe to be consumed by humans. Further research is needed to relate the results obtained from animal studies in order to assess the risk for human health.

Stevia rebaudiana Bertoni is a branched bushy shrub of the Asteraceae family, native to the Amambay region in the north east of Paraguay. Stevioside and rebaudioside A are the main sweetening compounds of interest, which are commercially produced by both chemical and physical processes. Stevia rebaudiana has a great potential as a new agricultural crop since consumer demand for health promoting foods is increasing, and it's leaves can be used as raw material for the extraction and production of functional food ingredients as well as source of carbohydrates, protein, crude fiber, minerals and other essential nutrients which are all valuable for human nutrition., ${ }^{5,6}$ Nowadays, due to the growth of the health food industry reduction of sucrose content of food products by full or partial replacement of sucrose using alternative sweeteners have become a viable option for producing low calorie/zero calorie foods. Low calorie food products of good quality can be made by incorporating combinations of non-caloric and carbohydrate sweeteners. ${ }^{7}$

Additionally, the previous literature findings were also reviewed which suggest that stevia could be used as a natural sweetener and a sugar replacer in different foods. Although, several researchers have claimed that stevia offers therapeutic benefits such as having antihyperglycemic, anti-hypertensive, anti-flammatory, anti-tumor, antidiarrheal, diuretic, and immunomodulatory effects; further study is needed to assess its toxicological effects on human health. It can be concluded that stevia is safe and can be used to promote and improve the human nutrition. ${ }^{5}$
Volume 2 Issue 3 - 2016

\author{
Mohammed Abdalbasit A Gasmalla ${ }^{1,2}$ \\ 'State Key Laboratory of Food Science \& Technology, Jiangnan \\ University, China \\ ${ }^{2}$ Department of Nutrition \& Food technology, Omdurman \\ Islamic University, Sudan
}

Correspondence: Mohammed Abdalbasit A Gasmalla, State Key Laboratory of Food Science \& Technology, Jiangnan University, 1800 Lihu Avenue, Wuxi 214122, Jiangsu, China, Tel +8615 I5220703 2, Email Gasmalla@jiangnan.edu.cn

Received: April 24, 2016| Published: May 04, 2016

\section{Acknowledgements}

None.

\section{Conflict of interest}

The author declares no conflict of interest.

\section{References}

1. Pól J, Hohnová B, Hyötyläinen T. Characterisation of Stevia Rebaudiana by comprehensive two-dimensional liquid chromatography time-of-flight mass spectrometry. J Chromatogr. 2007;1150(1-2):85-92.

2. Whysner J, Williams GM. Saccharin mechanistic data and risk assessment: urine composition, enhanced cell proliferation, and tumor promotion. Pharmacol Ther. 1996;71(1-2):225-252.

3. Dybing E. Development and implementation of the IPCS conceptual framework for evaluating mode of action of chemical carcinogens. Toxicology. 2002;181-182:121-125.

4. Authority EFS. Statement of EFSA on the scientific evaluation of two studies related to the safety of artificial sweeteners. EFSA. 2011;9(2):16.

5. Gasmalla MAA, Yang R, Hua X. Stevia rebaudiana Bertoni: An alternative sugar replacer and its application in food industry. Food Eng Rev. 2014;6(4):150-162.

6. Gasmalla MAA, Yang RA, Amadou I, et al. Nutritional composition of Stevia rebaudiana bertoni leaf: effect of drying method. Trop J Pharm Res. 2014;13(1):61-65.

7. Nabors LOB. Alternative sweeteners. 4th ed. USA: CRC Press; 2012. 587 p. 